\title{
Changes in the sublittoral benthic marine macroalgae in the polluted area of Abra de Bilbao and proximal coast (Northern Spain)
}

\author{
J. M. Gorostiaga*, I. Díez \\ Departamento de Biología Vegetal y Ecología, Facultad de Ciencias, Universidad País Vasco, Apdo. 644, \\ E-48080 Bilbao, Spain
}

\begin{abstract}
Sublittoral communities of macroalgae from the Abra de Bilbao and its surrounding area, which are affected by pollution, were studied in 1984 and 1992. Statistical cluster analysis applied to a network of 230 quadrats distributed along 7 transects resulted in 4 structural levels of community, depending on the pollution gradient. These levels are defined by algal cover, vertical layering and the predominant morphology of the species forming the community. Sedimentation, turbidity and water toxicity are the principal destabilizing factors among the phytobenthic communities. Species with simple morphology, mainly ceramiaceous algae (Ceramium diaphanum, Callithamnion neglectum, Antithamnionella spirographidis, Pterothamnion plumula, Anotrichium furcellatum, Compsothamnion thuyoides), sciaphilous (Mesophyllum lichenoides, Zanardinia prototypus, Rhodymenia coespitosella, Drachiella minuta, Erythroglossum laciniatum, Hypoglossum hypoglossoides) and those resistant to sedimentation (Pterosiphonia pennata and Champia parvula) are the most widely found species in severely polluted settings. Species such as Gelidium sesquipedale, Heterosiphonia plumosa, Calliblepharis ciliata. Halopitys incurvus, Dictyopteris polypodioides and Halurus equisetifolius are species typically found at the reference site and are good indicators of unchanged environments. Pterosiphonia complanata, abundant at the reference site, took the place of $G$. sesquipedale in those sites farthest from the source of pollution. Ordination and classification analyses detected changes in flora after 8 yr. pointing toward a recuperating process. The improvement consisted of an increase in algal cover along with a decrease in ceramiaceous algae and a greater complexity in community stratification. This recovery was directly related to a decrease in industrial activity and closure of the mineral washeries, as well as the establishment of urban wastewater-treatment plants for various municipalities.
\end{abstract}

KEY WORDS: Pollution - Sublittoral - Benthic macroalgae - Community parameters · Bioindicators . Recuperating process

\section{INTRODUCTION}

Benthic marine algae are good indicators of environmental changes caused by pollution of marine coastal waters. Attached algae, because of their sedentary nature, tend to integrate the effects of long-term exposure to adverse conditions. Studies on this subject have largely been carried out on the intertidal zone (Golubic 1970, Borowitzka 1972, Edwards 1972, 1975, Littler \& Murray 1975, Murray \& Littler 1978, May 1985, Brown

\footnotetext{
•E-mail: grpgogaj@lg.ehu.es
}

1990, Fairweather 1990) and thus information over the effects of pollution on sublittoral flora is more recent and scarce (Lundälv et al. 1986, Cormaci \& Furnari 1991). In general, pollution creates a degradation of flora resulting in an important decrease in species richness, an increase in abundance of a few ephemeral species with high reproductive capability and tolerance to pollution, and a simplification of community structure. However, there is little information about the inverse processes of recuperation. The purpose of this article is to document temporal (1984 to 1992) and spatial (17 km of coast) changes in the composition, cover, vertical stratification and nature of the sublittoral ben- 
thic vegetation in an area affected by severe pollution and which has seen a decrease in the pollution of its waters in the last decade.

\section{STUDY AREA}

The area studied was located in Northern Spain, in the mouth of the Nervion River (Abra de Bilbao) and its proximal coast $\left(43^{\circ} 20^{\prime} \mathrm{N}, 3^{\circ} 01^{\prime} \mathrm{W}\right)$ (Fig. 1). This area suffers from severe pollution as a result of industrial and urban dumping of non-treated wastes into the Nervión River over decades. Since the early 1980s there has been a decrease in the water pollution (Table 1) due to a recession in industrial activity and closure of the mineral washeries. This decrease has been even more noticeable since 1988, coinciding with the closure of 2 of the most important metallurgical plants and the initiation of primary wastewater treatment in various municipalities in the Bilbao metropolitan area.

Polluted waters of the Nervión River flow into the Abra de Bilbao and are carried eastward by currents influenced by the northwest wind. For this reason, 7 study sites were established along the $17 \mathrm{~km}$ coastline (Fig. 1). In the Abra proper there are 3 sites: Ciervana (Site 2) and Termica (Site 3), both semi-exposed to waves, and Arrigunaga (Site 4), which is exposed. The other 4 sites are along the open coast and are all very exposed to waves: Galea (Site 5), Meñakoz (Site 6) and Matxilando (Site 7) to the east and Muskiz (Site 1) to the west. Site 1 was considered the reference site due to low or non-existent exposure to the polluted

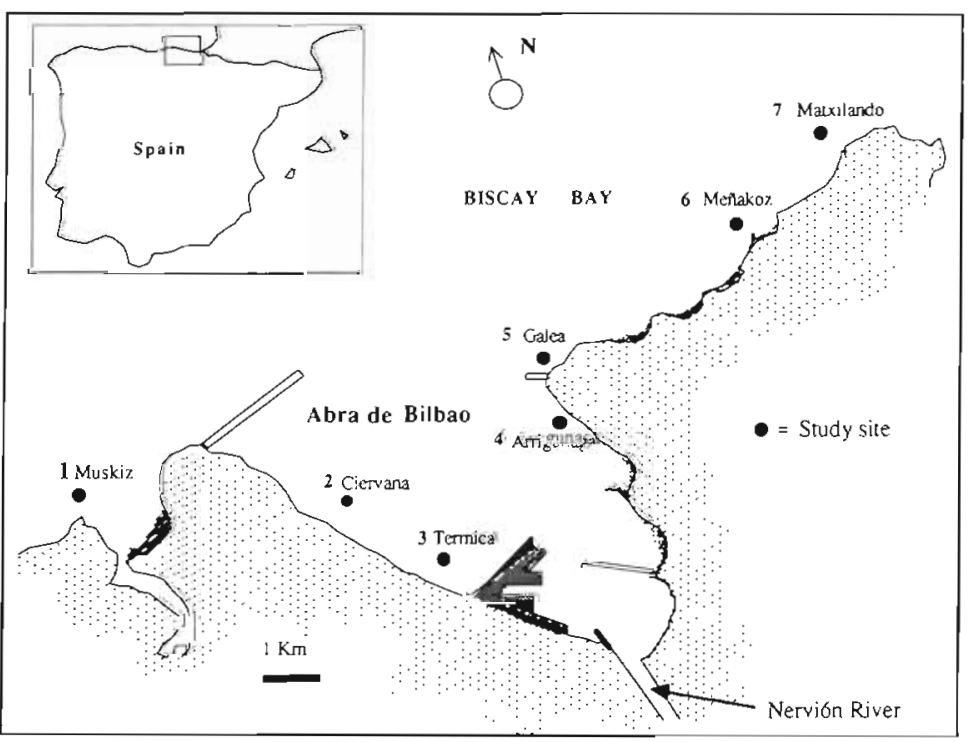

Fig. 1. Study area and sites in Northern Spain plume. The topography of Sites 2, 3, 5 and 6 was very regular, consisting of flat rocky shore platforms. Sites 4 and 7 had a slightly irregular topography with alternating spurs and depressions. Site 1 showed an abrupt topography as it was the elongation of a cliff. Salinity $(35 \%)$ and temperature $\left(12\right.$ and $22^{\circ} \mathrm{C}$ in February and August, respectively) showed no significant differences between the sites in the area studied. Only the site closest to the mouth of the Nervión River underwent small changes in salinity (33 to $35 \%$ ) and temperature (10 and $21^{\circ} \mathrm{C}$ for February and August).

\section{METHODS}

A $100 \mathrm{~m}$ long transect perpendicular to the coast was made at each site. The depth range covered by the transects varied according to the topography of the site. The transect began at a depth of 2 to $3 \mathrm{~m}$, and ended at depths between 6 and $10 \mathrm{~m}$ (except at Site 2 where the transect was as deep as $13 \mathrm{~m}$ ). An estimation of the cover ( $1 \mathrm{~m}^{2}$ quadrats) of the species was made at $3 \mathrm{~m}$ intervals according to the following scale (Braun-Blanquet $1932 / 1951):+(<1 \%) ; 1(1$ to $5 \%) ; 2$ (5 to $25 \%)$; $3(25$ to $50 \%) ; 4(50$ to $75 \%) ; 5(75$ to $100 \%)$. The sedimentation level (silt and sand) of the quadrats was also visually estimated using the following subjective scale (John 1968): 1 (little), 2 (moderate), 3 (heavy) and 4 (very heavy). Details concerning vertical layering of the community and a photographic register of each sample were also taken. The sampling was carried out in the summers of 1984 and 1992. Significance levels of the differences between cover means of both years were evaluated by Mann-Whitney U-test. Multivariate analyses of data were carried out using CANOCO (ter Braak 1988) and SYNTAX (Podani 1993) programs. Kent \& Coker (1992) and Jongman et al. (1987) report the description and application of multivariate analysis techniques for vegetation studies. Ordination analysis of quadrats using a linear model of indirect gradient (Principal Component Analysis) was carried out to register a possible ordination of the quadrats as a function of pollution as well as displacement of the quadrats in time and space. Finally, floristic differences between sites and different degrees of deterioration in the communities were determined using statistical cluster analysis for both years. The histogram transformation was applied to all percentage cover data. Chord distance was selected as the resemblance coefficient; the clustering strategy used was average linkage (UPGMA). Dendrograms of both years 
Table 1. Changes in volume discharged $(Q)$ and main pollutants in industrial and domestic effluents (information provided by the municipal authority for water supply and sewage 'Consorcio de Aguas del Gran Bilbao'

\begin{tabular}{|c|c|c|c|c|c|c|c|c|c|}
\hline \multicolumn{10}{|c|}{ Industrial sewage discharge } \\
\hline & $\begin{array}{c}Q \\
\left(m^{3} d^{-1}\right)\end{array}$ & $\begin{array}{l}\text { Acidity } \\
\left(\mathrm{kg} \mathrm{d}^{-1}\right)\end{array}$ & $\begin{array}{c}\text { Iron } \\
\left(\mathrm{kg} \mathrm{d}^{-1}\right)\end{array}$ & $\begin{array}{l}\text { Cadmium } \\
\left(\mathrm{kg} \mathrm{d}^{-1}\right)\end{array}$ & $\begin{array}{l}\text { Arsenic } \\
\left(\mathrm{kg} \mathrm{d}^{-1}\right)\end{array}$ & $\begin{array}{r}\text { Copper } \\
\left(\mathrm{kg} \mathrm{d}^{-1}\right)\end{array}$ & $\begin{array}{c}\text { Nickel } \\
\left(\mathrm{kg} \mathrm{d}^{-1}\right)\end{array}$ & $\begin{array}{c}\text { Lead } \\
\left(\mathrm{kg} \mathrm{d}^{-1}\right)\end{array}$ & $\begin{array}{c}\text { Zinc } \\
\left(\mathrm{kg} \mathrm{d}^{-1}\right)\end{array}$ \\
\hline 1984 & 67454 & 379308 & 53824 & 39.1 & 1121 & 345 & 34.3 & 189.6 & 3529 \\
\hline 1992 & 40374 & 54500 & 7977 & 0.9 & 0 & 59 & 17.3 & 58.6 & 238 \\
\hline \multicolumn{10}{|c|}{ Domestic sewage discharge } \\
\hline & $\begin{array}{c}Q \\
\left(\mathrm{~m}^{3} \mathrm{~d}^{-1}\right)\end{array}$ & $\begin{array}{c}\mathrm{BOD}_{5} \\
\left(\mathrm{~kg} \mathrm{~d}^{-1}\right)\end{array}$ & $\underset{\left(\mathrm{kg} \mathrm{d}^{-1}\right)}{\mathrm{COD}}$ & $\begin{array}{c}\text { TSS } \\
\left(\mathrm{kg} \mathrm{d}^{-1}\right)\end{array}$ & $\begin{array}{c}\mathrm{P}^{-\mathrm{PO}_{4}} \\
\left(\mathrm{~kg} \mathrm{~d}^{-1}\right)\end{array}$ & & & & \\
\hline 1984 & 250000 & 57500 & 100000 & 50000 & 2500 & & & & \\
\hline 1992 & 250000 & 50920 & 88557 & 37572 & 2306 & & & & \\
\hline
\end{tabular}

were compared in order to detect variations in relative distances between the sites.

\section{RESULTS}

From the 230 quadrats per year (Table 2), a total of 80 and 75 species of macrophytes (1984 and 1992, respectively) were recorded. The floristic composition was similar in both periods with a total of 63 species in common. Of the 13 species that appeared in the second year, only 3 (Saccorhiza polyschides, Spirulina sp. and Erythroglossum laciniatum) had a high frequency ( $\mathrm{n}=$ 32,29 and 20, respectively). In 1992, 17 species which had been observed in 1984 were not observed, Agloathamnion byssoides and Schyzimenia dubyii being the most noteworthy absences ( $n=36$ and 29 , respectively).

Mesophyllum lichenoides and Zanardinia prototypus were the species that had the greatest cover and were common to all sites (except Site 4) both years. Other species with an important cover were Pterosiphonia complanata, Callithamnion neglectum, Ceramium diaphanum, and Rhodymenia coespitosella, the last 3 being common at the sites closest to the source of pollution. Gelidium sesquipedale managed a high cover only at the reference site.

\section{Site ordination}

The quadrats are represented by the first and second axes of the PCA (Fig. 2). The first axis summarises $20.1 \%$ of the variability in the data set. Cumulative percentage variances of the second and third axes are $33.3 \%$ and $41 \%$, respectively. Ordination analysis was carried out for all the quadrats of 1984 and 1992 together (Figs. $2 \& 3$ ) noting the similarity in terms of composition and abundance of species. This reflects a circular arrangement of the quadrats according to the following sequence of sites: 4 (Arrigunaga), 2 and 3 (Ciervana and Termica), 5 (Galea), 6 (Meñakoz), 7 (Matxilando) and 1 (Muskiz). Site 4 as well as Site 1 (the closest and furthest sites from the pollution source, respectively) are markedly distanced from the other sites (Fig. 3). Each site is also slightly displaced with respect to itself in time. As a result, in 1992 Site 3 is closer to Site 5 , Site 5 to Site 6 , and Site 6 to Site 7. Site 7 did not show a significant change in its proximity to Site 1. An exception was observed in that the slight displacement of Sites 2 and 4 was opposite that of the other sites.

\section{Site classification}

In order to determine differences between sites with respect to composition and cover of species, population data was subjected to statistical cluster analysis for 1984 and 1992 (Figs. 4 \& 5, respectively). In both dendrograms, well-defined groups, clearly showing notable differences between quadrats, could be recognized (4 and 6 groups in 1984 and 1992, respectively).

In 1984, Group 4 (Fig. 4) differed the most from the rest, gathering all quadrats from Site 4 (Arrigunaga). These were characterised by a practical absence of flora due to a very heavy silt level (Table 3 ). The flora which was found there was characterised by extremely light species richness and cover, absence of stratification (including the crustose layer) and opportunistic species: Antithamnionella spirographidis, Pterothamnion plumula, Anotrichium furcellatum and Pterosiphonia pennata. In the 1992 dendrogram (Fig. 5), the same group (Group 4') maintained its differences from the rest of the groups. Newly appearing species were Asparagopsis armata (tetrasporophytic phase), Hypoglossum hypoglossoides and Erythroglossum laciniatum, the last 2 being exclusive to Site 4 . 
Table 2. Frequency (N) and average cover in \% (C) of species according to site and year. Species are listed in decreasing order of total average covers for 1984. The lower part of the table shows the total number of species per year and site. ${ }^{\prime} \cdot{ }^{\prime}$ and $\cdots$ indicate significance levels $p<0.05$, $p<0.01$ and $p<0.001$, respectuvely, for the Mann-Whitney U-test of differences between means. (Table continued across page)

\begin{tabular}{|c|c|c|c|c|}
\hline Species & & & Ral & \\
\hline & & 984 & $N^{199}$ & \\
\hline Mesophyllum lichenoides (J. Ellis \& Sol, Lemoine & 198 & 33,51 & 2024 & 4352 \\
\hline Zatlerdinia prototypus (Nardo) Nardo & 170 & 16.02 & $156 \mathrm{i}$ & 1882 \\
\hline Prerosiphoniá complanata (Clemente) Falkenb. & 101 & 8.85 & 1131 & 16.72 \\
\hline Ceramum diaphanum (Lightl), Roth & 92 & 4.97 & 26 & 0.31 \\
\hline Callithamnion neglectum (Feldm-Maz.) Bailes. \& Rom & 70 & 471 & 72 & 5.51 \\
\hline Geidium sesquipedale (Clemente) Thuret & 21 & 3.18 & 22 & 3.72 \\
\hline Rhodymenid coespitoselia LHardy-Halos & 118 & 2.30 & 39 & 0.63 \\
\hline Falkenbergia rufolanosa (Harv.) F.Schmitz" & 60 & 1.35 & 122 & 174 \\
\hline Chondria coerulescens [J. Agardh] Falkenb. & 57 & 3.31 & 24 & 0.24 \\
\hline Drachielláa minuta (Kylin) Maggs \& Hommersand & 32 & 0.89 & 88 & 080 \\
\hline mnionella spirographidis (Schiffn.) Woalast. & 74 & 0.85 & 22 & 005 \\
\hline C. Agardh & 45 & 0.64 & 10 & 0.12 \\
\hline Hoientiatocel & 67 & 0.58 & 33 & 0.22 \\
\hline Pterothaminion plumula (J. El & 60 & 0.47 & 13 & 0.06 \\
\hline Nitoptiyllum punctatum (Sta & 114 & 0.45 & 83 & 0.24 \\
\hline Dictyotó dichotoma (Huds.) J.V Lamour & 61 & 0.31 & 42 & 0.18 \\
\hline Calliblepharis ciliat & 24 & 0.31 & 26 & 0.30 \\
\hline Anotsichitum furcellatus & 19 & 0.30 & 13 & 0.03 \\
\hline Cryptopleura to & 25 & 0.25 & 36 & 0.55 \\
\hline Compsothamnion thuyoides (J.s Sm.) Nageli & 104 & 0.24 & 22 & 0.10 \\
\hline Desmarestia ligulata J.V Lamour & 27 & 0.23 & 18 & 0.07 \\
\hline Pterosiphonia pennata (C.Agardh) Falkenb. & 43 & 0.22 & 35 & 0.31 \\
\hline Champla parvula (C Agardhi Harv. & 65 & 0.21 & 9 & 003 \\
\hline Cladophorasp. & 80 & 0.18 & 105 & 0.36 \\
\hline Bonnemaisonia asparagoides (Woodw - C.Agardh & 28 & 017 & 3 & 0.01 \\
\hline Taond atomana (Woodw.) J.Agardh & 42 & 013 & 26 & 0.06 \\
\hline Gymnogongrus crenulatus (Turner) J.Agardh & 41 & 0.13 & 31 & 0.12 \\
\hline Plocamium cartilagineum (L.) P.S.Dixon & 40 & 0.12 & 72 & 0.69 \\
\hline Asparagopsis armata Harvey & 45 & 0.11 & 7 & 0.03 \\
\hline Ulva rigide C. Agardh & 39 & 0.11 & 22 & 0.07 \\
\hline Halopilys incurvus (Huds.) Batters & 16 & 0.11 & 2 & 0.00 \\
\hline Fhyllariopsis brewipes (C.Agardh) Henry \& South & 31 & 0.10 & 15 & 0.11 \\
\hline Hedarachinion ligulatum (Woodw.) Kutz & 20 & 0.10 & 1 & 0.00 \\
\hline Heterosiphonia plumosa (J.Ellis) Batters & 19 & 0.10 & 13 & 0.12 \\
\hline Polysiphonia polyspora (C.Agardh) J.Agardh & 10 & 0.10 & 2 & 0.00 \\
\hline 1) V Lamour & 25 & 0.09 & 16 & 0.07 \\
\hline Mont ex Hary. & 5 & 0.09 & 4 & 0.02 \\
\hline G) Grev. & 4 & 0.09 & 41 & 0.67 \\
\hline oides (Arm. ex Harv) Halos \& Rueness & 36 & 0.08 & - & 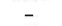 \\
\hline Corallina a & 28 & 0.08 & 29 & 0.11 \\
\hline Gelidium latifolitu & 25 & 0.08 & 1 & 0.00 \\
\hline Rhodymenia psel & 11 & 0.08 & 12 & 0.12 \\
\hline Bnopsidella halymeniae (Berthold) Feld & 7 & 0.08 & - & - \\
\hline Schizymensia dubys (Chauv, ex Duby) J.Agardh & 29 & 0.07 & - & - \\
\hline Callophyllis $\mathrm{sp}$. & 1 & 0.07 & - & - \\
\hline Apoglossum ruscifolium (Turnes) J Agardh & 26 & 0.06 & 7 & 0.03 \\
\hline Cryptonemis Lomation (Bertolini) J Agardh & 18 & 006 & 6 & 0.01 \\
\hline Pterocladia capillaceae (S.G.Gmelin) Bornet \& Thur & 15 & 0,04 & 1 & 0.00 \\
\hline Grateloupia dichotoma J Agardh & 14 & 0,04 & - & - \\
\hline Halurus equisetifolius (Lightt.) Kütz. & 14 & 0.04 & 19 & 0.07 \\
\hline Halopteris filicine (Gratel. Kutz. & 12 & 0.04 & 1 & 0.00 \\
\hline Sphond'ylothamnson multificium (Huds.) Nageli & 8 & 0.03 & 3 & 0.09 \\
\hline Acrosorium reptans (P.Crouan \& H.Crouan) Kylin & 7 & 0.03 & 1 & 0.00 \\
\hline Spatoglossum solieri (Chauv. ex Mont.) Kütz. & 5 & 0.03 & - & - \\
\hline Gigartina pisillata (S.G.Gmelin) Stackh. & 11 & 0.02 & 3 & 0.03 \\
\hline Codium decorticatum (Woodw) Howe & 9 & 0.02 & 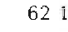 & 14.51 \\
\hline Grateloupld filicine (1, V Lamour) C Agardh & 8 & 0.02 & - & - \\
\hline sima Cocks ex Bornet & 8 & 0.02 & 4 & 0.01 \\
\hline Is lacimala (Hu & 7 & 0.02 & 30 & 0.55 \\
\hline Lomen & 7 & 0.02 & - & \\
\hline ardinil Kylin & 3 & 0.02 & 22 & 0.08 \\
\hline Cod: & 6 & 0.01 & 8 & 0.70 \\
\hline Jania & 5 & 0.01 & - & - \\
\hline Gelidism pusil & 4 & 0.01 & - & - \\
\hline $\begin{array}{l}\text { Bryopsis plum } \\
\text { Hoticustis par }\end{array}$ & 4 & 0.01 & 1 & 0.01 \\
\hline $\begin{array}{l}\text { Halleystis parv } \\
\text { Bornetia secun }\end{array}$ & 3 & 0.01 & 17 & 0.04 \\
\hline $\begin{array}{l}\text { Bornetic secunc } \\
\text { Cystosefsa bact }\end{array}$ & $\frac{1}{2}$ & 0.01 & 1 & 0.00 \\
\hline $\begin{array}{l}\text { P.Silva } \\
\text { Jol. }\end{array}$ & $\frac{2}{2}$ & 0.00 & 1 & 0.00 \\
\hline javerticil & $\begin{array}{l}2 \\
2\end{array}$ & 0.00 & - & $0 \overline{0}$ \\
\hline teedist(R) & $\frac{2}{2}$ & 0,000 & 1 & 0.01 \\
\hline & $\frac{2}{1}$ & $\begin{array}{l}0.00 \\
0.00\end{array}$ & 7 & 0.04 \\
\hline Ides (Stackh.) Collins \& Herv. & 1 & 0. & 18 & 0.08 \\
\hline Halopteris scopara (L. & 1 & 0.00 & - & - \\
\hline Gracilarta bursa-pastoris IS.G.Gmelin) P C. Silva & 1 & 0.00 & - & \\
\hline Gymogongros patens J.Agd! & 1 & 0.00 & 7 & 0.01 \\
\hline (Harv.) Collins ex W.R. & 1 & 0.00 & - & - \\
\hline a (J. Agardh) J.Agardh & 1 & 0.00 & - & - \\
\hline Nace: & $!$ & 0.00 & - & - \\
\hline Peysso & 1 & 0.00 & 1 & 0.00 \\
\hline Saccorhiza polyschides (Lightf.) Batters & - & $=$ & 32 & 206 \\
\hline & - & - & 29 & \\
\hline & - & - & 9 & \\
\hline & - & - & 3 & 0.07 \\
\hline Ery: & - & - & 20 & 0.05 \\
\hline Ulvă & 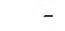 & - & 7 & 0.0 \\
\hline Pes & - & - & 2 & 0.1 \\
\hline & - & - & 4 & 0.1 \\
\hline liss Du & - & - & 2 & 00 \\
\hline & - & - & 2 & 0.00 \\
\hline nosporiums flexuosumi & $\begin{array}{l}- \\
-\end{array}$ & - & $i$ & $\begin{array}{l}0.00 \\
0.00\end{array}$ \\
\hline TOTAL & 41 & 85 & 1961 & 116 \\
\hline Sprecies richness & & 30) & 75 & \\
\hline
\end{tabular}


Table 2 (contınued)

\begin{tabular}{|c|c|c|}
\hline Spuen' & & 4: A \\
\hline & 19 & 984 \\
\hline 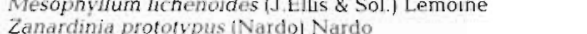 & 1 & 0.01 \\
\hline $\begin{array}{l}\text { Zanardinia prototypus (Nardol Nardo } \\
\text { Pterosiotionia complantä (Clemente) F }\end{array}$ & - & - \\
\hline 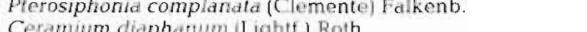 & - & - \\
\hline Ceramium diaphanum (Lightf) Roth & - & - \\
\hline Callithamnion neglectum (Feldm.-Maz) Balles. \& Rom. & - & - \\
\hline Gelsdium sesquipedate (Clemente) Thuiet & - & $\therefore$ \\
\hline Rhodymenia coespitosella L'Hardy -Halos & 1 & 0.01 \\
\hline Falkenbergia rufolanosa ! Harv F Schmita" & - & - \\
\hline Chondria coerulescens (J. Agardhi Falkenb & - & - \\
\hline Drachiella minuta (Kylin) Maggs \& Homnersand & & \\
\hline Antithamnionella spirographidis iSchiffn.) Woolast. & 7 & 0.12 \\
\hline Cladostephus spongiosus (thuds) C.Agardh & 1 & 0.01 \\
\hline Haematocelis rubens I. Agardh" & 3 & 005 \\
\hline Pterothamnion piumula (f. Ellis) Nageli & 2 & 003 \\
\hline Nitophyllum punctatum (Stackh.) Gres. & - & - \\
\hline Dictyola dichutoma (Huds.) J.V.Lamour & - & - \\
\hline Callblephatis cilidid (Huds.) Küz. & - & - \\
\hline Anotrichum furcellatum (1. Agardh) Baldock & 3 & 0.05 \\
\hline Cryptopleura ramosa (Huds) Kylin ex Newton & & \\
\hline Compsothamnion thuyoides (J.s Sn ) Nageli & 3 & 0.05 \\
\hline Desmarestia Ligulata J V. Lamour & - & \\
\hline Pterosiphonia penndta (C Agardin) Falkenb & 5 & 0.08 \\
\hline Champia parvisla (C.Agardhi) Haiv. & - & - \\
\hline Cladophora sp & 1 & 0.02 \\
\hline Bonnemaisonia asparagoides (Woodw. C. Agardh & - & - \\
\hline Taonia atomania (Woodw.) J Agardh & - & - \\
\hline Gymnogongrus crenulatus (Turner) J.Agardh & - & - \\
\hline Plocamum carthogineum (L).) P.S Dixon & - & - \\
\hline Asparagopsis armata Harvey & - & - \\
\hline Ulva rigida C.Agardh & - & - \\
\hline Halopitys incurvus (Huds.) Batters & - & - \\
\hline Phyllariopsis brevipes (C Ágardh) Henry \& South & - & -- \\
\hline Halärachnion Ligulatum (Woodw.) Kütz & - & - \\
\hline Heterosiphona plumosa (J Ellis) Batters & - & - \\
\hline Polysiphonia polyspora (C.Agardh) J.Agardh & - & - \\
\hline Dictyopteris polypodioides (D.C.) J.V Lamour & - & - \\
\hline Stenogramine internupta (C.Agardh) Mont. ex Hary. & - & - \\
\hline Microcladia glandulosa (Sol. ex Turner) Grev. & - & - \\
\hline Aglaothammion byssoides (Arn. ex Harv.) Halos $\&$ & - & - \\
\hline Corallina onicnalis $\mathrm{L}$ & - & - \\
\hline Gelidium lathollum (Grev) Borne & - & - \\
\hline Rhodymenia pseuclopalmata (J.V.Lamour) P.C.Silva & - & - \\
\hline Bropsidella halymeriae (Berthold) Feldmann & - & - \\
\hline Schizymena dubyi (Chatw ex Duby) J.Agardh & - & - \\
\hline Callophyllis sp. & - & - \\
\hline Apoglossum ruscifolium (Turner) J Agardh & & - \\
\hline Cyptoremia lomation (Bertolini) J Agardh & - & - \\
\hline Pterocladia capillacede (S.G.CGmelin) Bomet \& Thur. & - & - \\
\hline Grateloupia dichrotoma J A gardh & - & - \\
\hline Halurus equisetitolus (Lightf) Kütz & - & - \\
\hline Halopteris filicind IGratel i kutz & - & - \\
\hline Sphondylothamnion multifid um (thuds i Nägeli & - & - \\
\hline Acrosorium reptans (P.Crouan \& H Crouan) Kylin' & 2 & 0.03 \\
\hline Spatoglossum solieri iChauv ex Mont I Kulz. & - & - \\
\hline Gigartina pistlllata (S.G.Gmelin) Stackh. & - & - \\
\hline Codium decorticatum (Woodw, Howe & - & - \\
\hline Grateloupia filicina (I V.Lamour) C Agardh & - & - \\
\hline Polysiphonid foetidissima Cocks ex Bornet & - & - \\
\hline Callophylis lacimata (Huds.) Kutz. & - & - \\
\hline Lomentara clavellosa (Turner) Gaillon & - & - \\
\hline Acrosorium venulosum (Zanardini) Kylin & - & - \\
\hline Codium adtiaerens C.Agardh & - & - \\
\hline Jania cormiculata (L.) J.V Lamour & - & - \\
\hline Gelidium pusilum (Stackh.) Le Jol. & - & - \\
\hline Bryopsis plumosa if & 1 & 0.02 \\
\hline Hahcystis parvula Schmidt ex Murray ${ }^{f}$ & - & - \\
\hline Bornetia secundiflora (J Agardhi Thur. & - & - \\
\hline Cystoseira baccata [S G. Cimelin) P.C. Silva & - & - \\
\hline Dudresndya verticillata (With) Le Jol & - & - \\
\hline Gigartina teediu (Roth) J.V.Lamour & - & - \\
\hline Scinaia furcellata (Turner) J.Agardh & - & - \\
\hline Codium fragie ISuringari Hanot & - & - \\
\hline Hypoglossum bypoglossoides (Stackhi) Collins \& Herv. & : & 002 \\
\hline Halopteris scoparia (L) I Sauv. & - & - \\
\hline Gracilaria bursa-pastoris (S G. Gmelin) P.C Silva & - & - \\
\hline Gymnogongrus patens J Ayardh & - & - \\
\hline Lomentaria orcadensis ( Harv ) Collins ex W. R. Taylor & - & - \\
\hline Meredithia microphylls [] Agardh) J Agardh. & - & - \\
\hline Naccaria wiggi (Tumer) Endil. & - & - \\
\hline 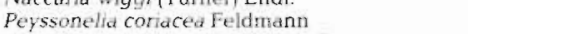 & - & - \\
\hline Saccorhiza polyschides (Lightf.) Batters & - & - \\
\hline Spirulina sp & - & 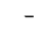 \\
\hline ens (L.) J.V.Lamo & - & - \\
\hline & - & - \\
\hline aggs \& & - & - \\
\hline Ulva olivascens P. A.Dang. & - & - \\
\hline Peyssonella sp. & - & - \\
\hline Rhodymenia sp. & - & - \\
\hline Ceramum culiatum Ellisj Ducluz. & - & - \\
\hline Kallymenia reniformis iTurner) J Agardh & - & \\
\hline $\begin{array}{l}\text { Halymenia latifolia P. Crouan \& H.Crouan ex Kütz. } \\
\text { Pleonosporium flexuosim (C Aqardh) Bomet }\end{array}$ & - & $\overline{-}$ \\
\hline TOTAL & & 050 \\
\hline Species richness & 13 & \\
\hline
\end{tabular}




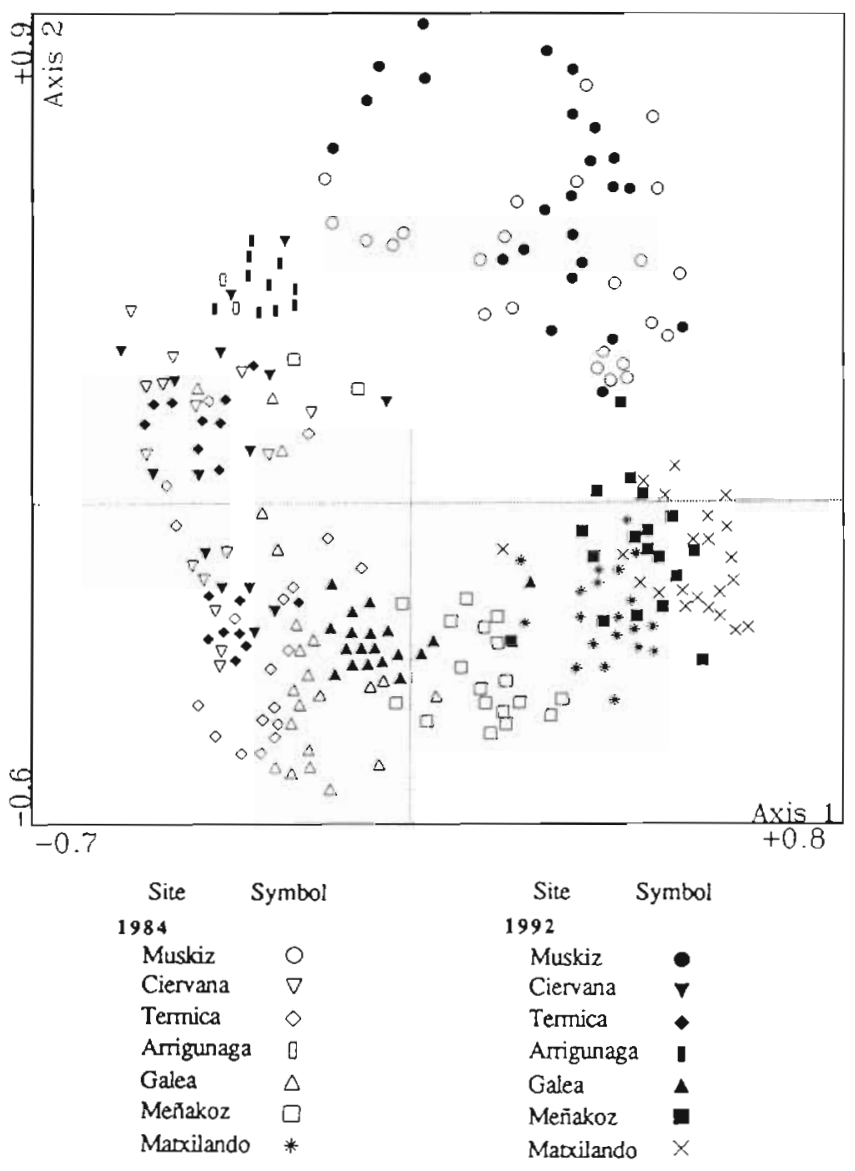

Fig. 2. Ordination plot for the first 2 axes of principal component analysis (PCA) of the quadrat data

In 1984, Group 2 (Fig. 4) combined the quadrats from Sites 3 (Termica), 2 (Ciervana) and 5 (Galea). Corresponding to the quadrats of each site were 3 subgroups $(2 \mathrm{~A}, 2 \mathrm{~B}, 2 \mathrm{C})$ and, along with these, a small group of shallow-depth quadrats in Sites 2 and 3 (2D). The 3 subgroups represented a degraded and homogeneous flora which supported heavy silt levels (Table 3 ). They were characterised by moderate cover and a strongly developed crustose layer of Mesophyllum lichenoides and Zanardinia prototypus with a poorly developed understory layer. The shallow-depth quadrats of Sites 2 and 3 were characterised by a poor crustose layer, from which $Z$. prototypus was absent. The group had an understory layer mainly composed of species with simple morphology such as Callithamnion neglectum. Ceramium diaphanum, Anotrichium furcellatum, Antithamnionella spirographidis, Pterothamnion plumula and Compsothamnion thuyoides. Species with complex morphology were basically represented by Rhodymenia coespitosella, which was abundant in rocky juts. This type of vegetation lacks canopy. In the 1992 dendrogram (Fig. 5), Subgroups $2 \mathrm{~A}$ and $2 \mathrm{~B}$ were fused in Subgroup $2 \mathrm{~A}^{\prime} \mathrm{B}^{\prime}$, Subgroup $2 \mathrm{C}$ was maintained $\left(2 \mathrm{C}^{\prime}\right)$ and for the conjunction of the shallow-depth quadrats from Sites 2 and 3(2D), a new group was formed (2D'). The most notable changes were the significant increase in the crustose layer at the Site 5 (from 70 to $145 \%$ ) and the significant decrease in species such as Rhodymenia coespitosella and other ceramiaceous algae $(C$. diaphanum, A. spirographidis, P. plumula) at the 3 sites. The appearance of the new group (2D') is explained by the practical absence of a crustose layer in shallow-depth quadrats and by proliferation of new species such as Codium adhaerens, Codium decorticatum and Spirulina sp. instead of the ceramiaceous algae. Absence of the crustose layer was basically due to substrate occupation by the bivalve Mytllus edulis.

In 1984, Group 3 (Fig. 4) gathered all quadrats from Sites 6 (Meñakoz) and 7 (Matxilando). A subgroup ( $3 \mathrm{~A}$ and $3 \mathrm{~B}$, respectively) corresponded to each site. The flora was degraded and more heterogeneous than that in Group 2, having heavy sand and moderate silt levels (Table 3 ). Cover was moderate with well-developed crustose (Mesophyllum lichenoides and Zanardinia prototypus) and understory (Pterosiphonia complanata, Rhodymenia coespitosella, Falkenbergia rufolanosa, Chondria coerulescens, Callophyllis laciniata,

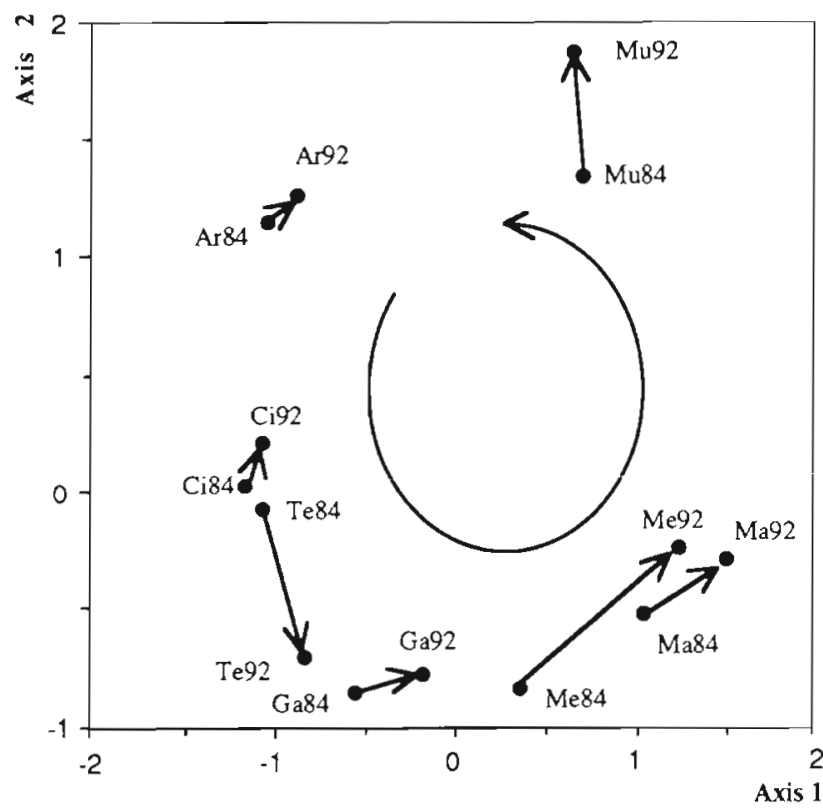

Fig. 3. Plot of the site group average scores for the first and second PCA axes. Arrows show displacement from 1984 to 1992. Mu: Site 1, Ci: Site 2, Te: Site 3, Ar: Site 4, Ga: Site 5, Me: Site 6 and Ma: Site 7 


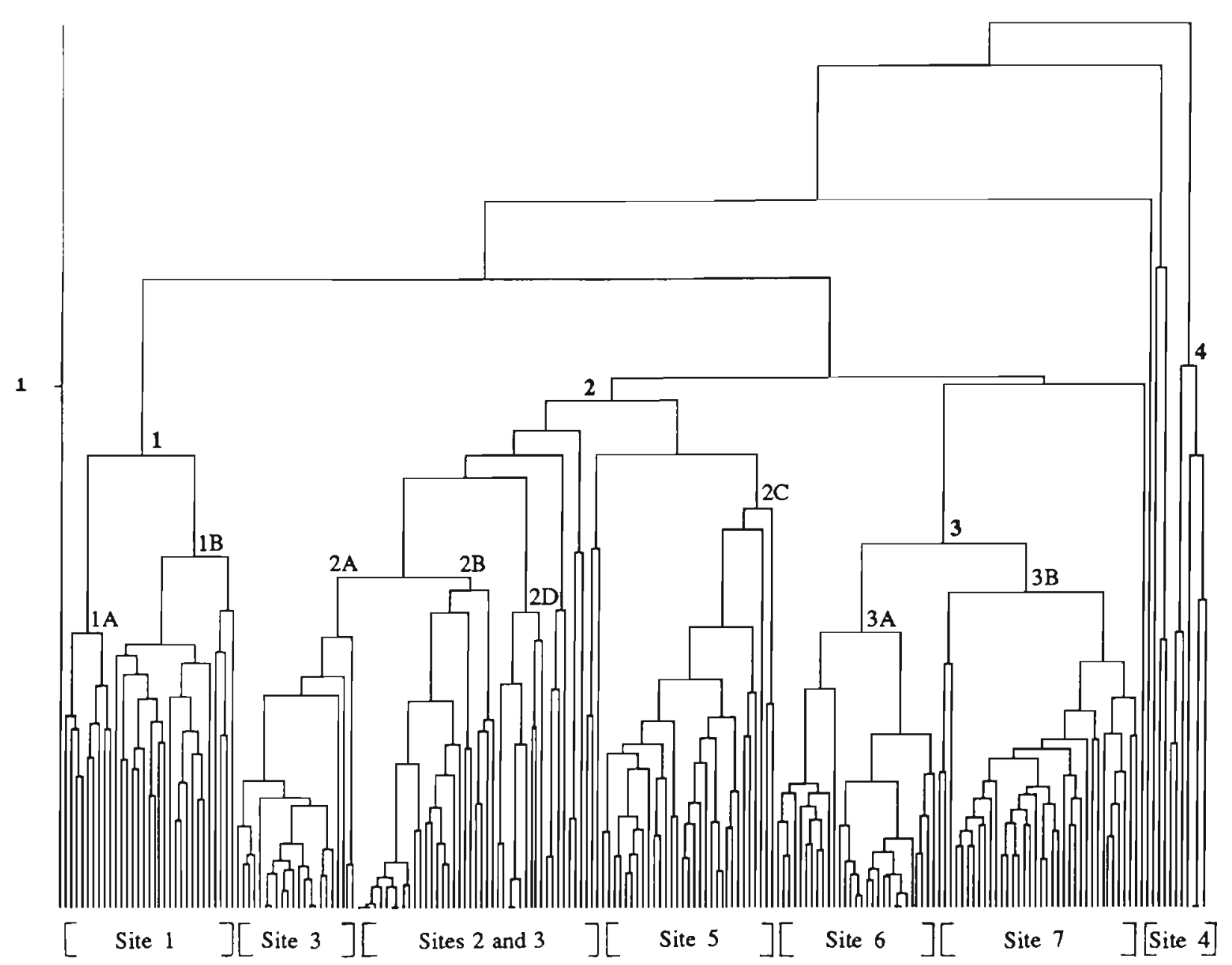

Fig. 4. Cluster analysis of all quadrats for 1984 using chord distance as resemblance coefficient and average linkage (UPGMA) as clustering method. This presentation shows 4 groups (1 to 4 ) which represent 4 levels of degradation in the vegetation

Ceramium diaphanum, Drachiella minuta, Nitophyllum punctatumi layers. P. complanata was the dominant species in the understory layer ( $36 \%$ cover). This type of vegetation lacks a canopy. In the 1992 dendrogram (Fig. 5), the same group was maintained $\left(3^{\prime}\right)$, although with a greater degree of homogeneity due to the fusion of the 2 subgroups. The most notable changes were the increase in cover (from moderate to high) due to the appearance of a dense canopy of the annual species Codium decorticatum at Site 6 (partially developing at Site 7) and the increase of the crustose and understory layers at Site 7. Understory layer species such as $P$. complanata, $D$. minuta, C. laciniata and Plocamium cartilagineum showed significant increases in cover to the detriment of other species (C. diaphanum, Antithamnionella spirographidis, R. coespitosella, C. coerulescens, Cladostephus spongiosus and $N$. punctatum). Gelidium sesquipedale, a perennial species which formed a canopy at the reference site, appeared for the first time at Site 7 in 1992 .

In 1984, Group 1 (Fig. 4) was composed of the quadrats from Site 1 (reference site). This was the only group whose vegetation did not alter. There were 2 subgroups ( $1 \mathrm{~A}$ and $1 \mathrm{~B}$ ) corresponding to the communities of Gelidium sesquipedale and Pterosiphonia complanata, which supported slight and moderate sand levels, respectively (Table 3 ). Both had a well-developed crustose layer of Mesophyllum lichenoides (the cover of Zanardinia prototypus was very thin). The poor understory layer of the $G$. sesquipedale community was basically formed by Pterosiphonia complanata, Rhodymenia pseudopalmata and Cryptopleura ramosa. G. sesquipedale formed a dense canopy, over which Plocamium cartilagineum constituted a poor epiphytic layer. The understory layer of the Pterosiphonia complanata community became highly developed and was basi- 


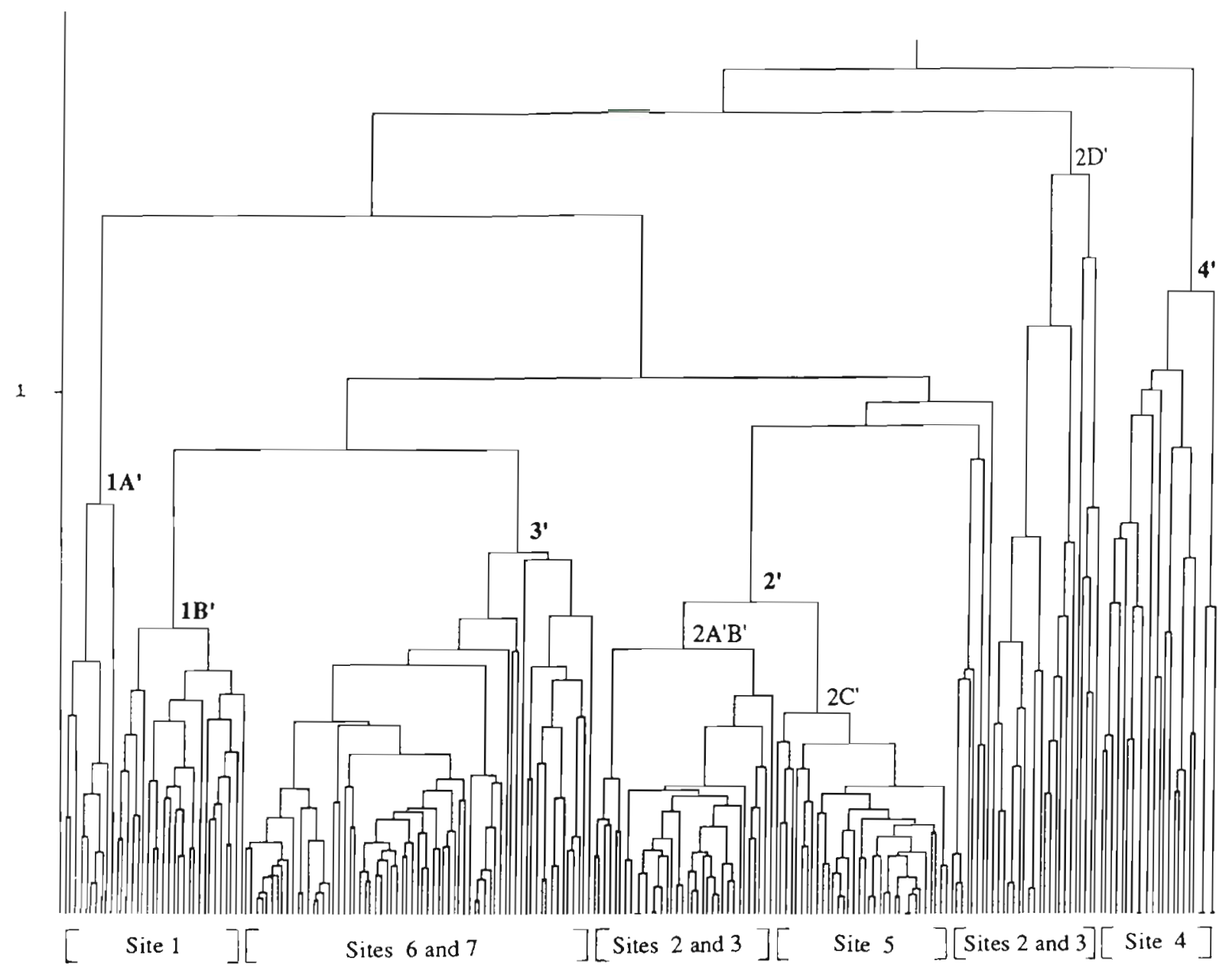

Fig. 5. Cluster analysis of all quadrats for 1992. Dendrogram displays 6 groups $\left(1 \mathrm{~A}^{\prime}, 1 \mathrm{~B}^{\prime}, 3^{\prime}, 2^{\prime}, 2 \mathrm{D}^{\prime}\right.$ and $\left.4^{\prime}\right)$ which correspond to 4 levels of degradation just as in 1984

cally made up of $P$. complanata, Falkenbergia rufolanosa, Desmarestia ligulata, Calliblepharis ciliata, Cryptopleura ramosa, Pterosiphonia pennata, Halopitys incurvus, Heterosiphonia plumosa and Plocamium cartilagineum. In the 1992 dendrogram (Fig. 5), a separation of this group into 2 groups (1 $\mathrm{A}^{\prime}$ and $1 \mathrm{~B}^{\prime}$ ) can be observed. Thus the community of $P$. complanata became combined with Group 3'. The most notable changes were the increase in cover from high to very high) in the community of $G$. sesquipedale due to the appearance of an upper canopy of the annual species Saccorhiza polyschides and the increase of crustose and understory layers of the $P$. complanata community.

\section{DISCUSSION}

The circular disposition of the quadrats detected in the PCA correspond to a sequence of sites according to a gradient of decreasing pollution from Sites 4 to 1
(Figs. $2 \& 3$ ). The discontinuity observed between Sites 4 and 2 and between Sites 1 and 7 is due to the absence of a gradual transition of the flora. The displacement of the quadrats recorded from 1984 to 1992 in the direction of the reference site (Fig. 3) represents an improvement of the sites. The slight displacements of Sites 2 (Ciervana) and 4 (Arrigunaga) in the opposite direction occurred for different reasons. Site 2 underwent a regression as the result of the negative influence of construction in the enlarging of Port of Ciervana. This construction increased the levels of sedimentation and turbidity in the water column. The regression of Site 4 was of a more mathematical nature, being due to the unequal number of quadrats computed for both years ( 5 and 23 samples with flora for 1984 and 1992, respectively).

From the classification analysis, 4 community structural levels in the area studied can be distinguished for both years. These reflect 4 different degrees of pollution, which are delineated here in decreasing order (Table 3). 
Table 3. Vertical layering cover (\%), percentage cover (\%) of morphologically simple species, species richness (species number) and sedimentation estimates (see 'Methods') for the different degradation levels and sites (values are averages for each quadrat). G.S.: Gelidium sesquipedale community; P.C.. Pterosiphonia complanata community

\begin{tabular}{|c|c|c|c|c|c|c|c|c|c|c|c|c|c|c|c|c|}
\hline \multirow{3}{*}{$\begin{array}{r}\text { Study site: } \\
\text { Degradation level: }\end{array}$} & \multirow{2}{*}{\multicolumn{2}{|c|}{$\begin{array}{l}\text { Site } 4 \\
\text { Level } 1\end{array}$}} & \multicolumn{2}{|c|}{ Site 2} & \multicolumn{2}{|c|}{$\begin{array}{l}\text { Site } 3 \\
\text { Level } 2\end{array}$} & \multicolumn{2}{|c|}{ Site 5} & \multicolumn{2}{|c|}{ Site 6} & \multicolumn{2}{|c|}{ Site 7} & \multicolumn{4}{|c|}{ Site 1} \\
\hline & & & & & & & & & & & & & & G.S. & & $\mathrm{C}$ \\
\hline & 1984 & 1992 & 1984 & 1992 & 1984 & 1992 & 1984 & 1992 & 1984 & 1992 & 1984 & 1992 & 1984 & 1992 & 1984 & 1992 \\
\hline Crustose layer cover & 0.1 & 0.2 & 60 & 44 & 76 & 67 & 70 & 145 & 59 & 39 & 44 & 75 & 56 & 60 & 29 & 53 \\
\hline Understory layer cover & 0.4 & 3.1 & 44 & 29 & 29 & 26 & 21 & 4 & 41 & 37 & 46 & 63 & 9 & 35.5 & 56 & 86 \\
\hline Canopy cover & - & - & - & - & - & - & - & - & - & 78 & - & 19 & 68 & 92.5 & - & - \\
\hline Epiphytic layer cover & - & - & - & - & - & - & - & - & - & - & - & - & - & 4 & - & - \\
\hline Total cover & 0.5 & 3.3 & 104 & 73 & 105 & 93 & 91 & 149 & 100 & 154 & 90 & 157 & 133 & 192 & 85 & 139 \\
\hline $\begin{array}{l}\text { Percentage cover of } \\
\text { morphologically } \\
\text { simple species }\end{array}$ & 96 & 83 & 78 & 83 & 87 & 80 & 76 & 66 & 38 & 73 & 18 & 42 & 1.2 & 1.7 & 14.9 & 4.4 \\
\hline Species richness & 1.03 & 4.6 & 5.4 & 5.2 & 10 & 8.8 & 10 & 8.1 & 11 & 10 & 20 & 12 & 11 & 9 & 19 & 11 \\
\hline Sedimentation level & 4 & 4 & 3.53 & 3.59 & 3.29 & 3.26 & 2.62 & 2.97 & 3.71 & 2.91 & 3.56 & 2.41 & 0.44 & 1.2 & 2.41 & 1.96 \\
\hline
\end{tabular}

Level 1: Extremely low species richness and algal cover, absence of stratification (including the crustose layer) and opportunistic nature of species. Smothering of the rocky bottom by sediments and extreme turbidity of the water cause the practical disappearance of the flora while favouring faunal development, e.g. species such as Actinothoe sphyrodeta and Phoronis hippocrepia. Proliferation of invertebrates in polluted environments has recently been noted by various authors (Littler \& Murray 1975. Klavestad 1978, Munda 1980, Brown et al. 1990). In these environments the only species that can survive are those resistant to high sedimentation, such as Pterosiphonia pennata, sciaphilous algae, such as Hypoglossum hypoglossoides and Erythroglossum laciniatum, and those with simple morphology (fundamentally ceramiaceous algae). Site 4 belongs to Level 1 (dendrogram Groups 4 and 4'), indicating chronic, direct impact of the polluted plume. The slight increase detected in species number and their cover between years is an indication of slight recuperation; however, it is still insufficient for the site to resemble Level 2.

Level 2: Moderate algal cover, a strongly developed crustose layer, a poor understory layer with a high percentage of opportunistic species and absence of canopy. The high water turbidity and the partial smothering of the bottom by sediments coming from the polluted plume give rise to a very poor flora. The strong development of the crustose species Mesophyllum lichenoides and Zanardinia prototypus is encouraged by the absence of a canopy, a weak development of the understory layer, scarce illumination and its resistance to sedimentation. Both species are sciaphilous (Boudouresque 1985, Afonso-Carrillo et al. 1992). Sites 2, 3 and 5 (Groups 2 and 2 ' of the dendrograms) belong to this level as they are frequently affected by the polluted plume and are therefore subjected to heavy pollution. The significant increase in crustose layer cover and the decrease in algal cover of simple morphology between 1984 and 1992 at Site 5 were favourable changes. Also, the noteworthy decrease in ceramiaceous algae cover, partially substituted by Codium adhaerens, Codium decorticatum, Cladophora pellucida and Corallina officinalis, was a sign of improvement at Site 3. According to our observations of the lower littoral zone, $C$. officinalis is the substitution stage for ceramiaceous algae when there is an improvement in water quality. Site 2 underwent a notable setback in reduction of crustose and understory layers due to construction activitities in the enlargement of Port of Ciervana.

Level 3: Moderate-high algal cover, moderate development of crustose and understory layers, moderate percentage of opportunistic species and absence of canopy (or presence of an annual canopy). The understory layer develops fully to the detriment of the crustose species. Site improvement is confirmed by an increase in species of complex morphology in the understory layer (to the detriment of the ceramiaceous algae), along with development of an annual canopy of Codium decorticatum and the appearance of fronds of Gelidium sesquipedale. However, the absence of other species from the understory layer of non-polluted environments (i.e. Level 4) and the absence of a perennial canopy indicate a state of degradation. Sites 6 and 7 (Groups 3 and $3^{\prime}$ of the dendrograms) belong to Level 3 because they are often affected by the polluted plume, a situation which is due to the dynamics of the coastal currents. Also, both sites suffer the negative effects of various local effluents from small urban centres.

Level 4: Very high algal cover, moderately developed crustose layer, poor understory layer (when there is no canopy this last is fully developed) and a low per- 
centage of opportunistic species. Generally there is a canopy equipped with an epiphytic layer. Gelidium sesquipedale is an example of a mature, stable community which efficiently uses its space through its complex vertical layering. In this case, the dominance of the canopy is favoured over the other layers. As sedimentation progresses with depth and in the depressions, Pterosiphonia complanata, a more competitive species in these environments, replaces $G$. sesquipedale. In this case, the vegetation presents a simpler stratification and no canopy or epiphytic layer, thus favouring the development of an understory layer. Due to their abundance, the following species are also indicative of Level 4: Calliblepharis ciliata, Heterosiphonia plumosa, Dictyopteris polypodioides, Halopitys incurvus and Halurus equisetifolius. Site 1, the reference site not affected by the polluted plume, belongs to this level (Groups 1 and $1^{\prime}$ of the dendrograms). Most of the species mentioned for Site 1 are rare or absent at the rest of the sites. The flora remained fairly stable between the 2 years although there were some significant increases and decreases in cover of various species (Table 2). Sacchorhiza polyschides was the most noteworthy case. This species undergoes annual advances and setbacks all along the Basque coast due to temperature changes (Borja \& Gorostiaga 1990). However, significant changes in abundance of species such as Pterosiphonia complanata, Mesophyllum lichenoides and Cryptopleura ramosa are largely explained by the decrease in sandy sources.

All of these changes basically took place between 1988 and 1992, when there was a major decrease in the amount of contaminants being dumped into the waters.

In summary, decrease in pollution leads to a decrease in sedimentation, turbidity and toxicity of the water, factors which negatively affect algal development (Grigg \& Kiwala 1970, Hagmeier 1971, Rai et al. 1981). In these unstable environments the community responds by simplifying its structure: reducing the number of layers (Belsher 1974, Littler \& Murray 1975, Cormaci et al. 1985, Brown et al. 1990), reducing vegetal cover (Belsher 1974, Murray \& Littler 1977, Cormaci \& Furnari 1991), and allowing a proliferation of opportunistic species with simple morphology (Murray \& Littler 1978, Lundälv et al. 1986), especially ceramiaceous algae (Belsher \& Boudouresque 1976, Ballesteros \& Pérez-Vallmitjana 1984, Lundälv et al. 1986). There is also proliferation of sciaphilous (Cormaci et al. 1992) and sedimentation-resistant species. The crustose layer, made up of species having these characteristics, shows strong development in polluted environments (Cormaci \& Furnari 1991). Several authors have demonstrated a reduction of species richness in polluted environments (Borowitzka 1972, May 1985, Murray \& Littler 1977 ); this was also the case in the most degraded sites of the study area. However, in the sites not affected by severe pollution the number of species found is the same as the number in clean environments. Thus the previously described parameters are necessary to evaluate the deterioration level of the environment.

Finally, we can confirm that the transect technique was very adequate for monitoring as the changes produced were well reflected. However, the occasional difficulties in re-locating the transects (with the same orientation, bathymetrics and type of substrate) along with the contagious pattern of spatial distribution of several species (those with strong vegetative growth) indicate that the use of fixed quadrats is ideal when carrying out maximum accuracy monitoring.

Acknowledgements. This work is a result of research sponsored by the municipal authority for water supply and sewage 'Consorcio de Aguas del Gran Bilbao'.

\section{LITERATURE CITED}

Afonso-Carrillo J, Pinedo S, Elejabeitia Y (1992) Notes on the benthic marine algae of the Canary Islands. Cryptogam Algol 13:281-290

Ballesteros E, Pérez-Vallmitjana M, Zabala M (1984) Aproximación al conocimiento de las comunidades algales de la zona infralitoral superior en la costa catalana. Collectanea bot (Barc) 15:69-100

Belsher T (1974) Séquence des effets d'un égoût urbain, en fonction de l'éloignement de la source de pollution, sur les peuplements photophiles de mode battu (fraction algale); premiers résultats. Bull Soc Phycol Fr 19:158-163

Belsher T, Boudouresque CF (1976) L'impact de la pollution sur la fraction algale des peuplements benthiques de Méditerranée. In: Atti tavola rotonda internazionale 'La biologia marina per la difesa e per la produttività del mare' Stem-Mucchi, Modena, p 215-260

Borja A, Gorostiaga JM 1990 Distribución geográfica de Saccorhiza polyschides (Light.) Batt. en la costa vasca. Su posible relación con la temperatura. In: Gallego L (ed) Bentos 6. Actas VI simposium Ibérico estudios bentos marino. Bilbilis, Palma de Mallorca, p 1-8

Borowitzka MA (1972) Intertidal algal species diversity and the effect of pollution. Aust $\mathrm{J}$ mar Freshwat Res 23:73-84

Boudouresque CF (1985) Groupes écologiques d'algues marnes et phytocenoses benthiques en Méditerranée nord-occidentale: une revue. G Bot Ital 118:7-42

Braun-Blanquet $J$ (1932) Plant sociology: the study of plant communities. McGraw Hill, New York

Brown VB, Davies SA, Synnot RN (1990) Long-term monitoring of the effects of treated sewage effluent on the intertidal macroalgal community near Cape Schanck, Victoria, Australia. Botanica mar 33:85-98

Cormaci M, Furnari G (1991) Phytobenthic communities as monitor of the enviromental conditions of the Brindisi coast-line. Oebalia 17:177-198

Cormaci M, Furnari G, Giaccone G, Colonna P, Mannino AM (1985) Metodo sinecologico per la valutazione degli apporti inquinanti nella rada di Augusta (Siracusa). Boll Accad Gioenia Sci Nat 18:829-850 
Cormaci M, Furnari G Scammacca B, Serio D, Pizzuto F, Alongi G, Dinaro R (1992) La vegetazione marina di substrato duro dell'isola di Salina (Isole Eolie). Boll Accad Gioenia Sci Nat 25:115-144

Edwards P (1972) Benthic algae in polluted estuaries. Mar Pollut Bull 3:55-60

Edwards P (1975) An assessment of possible pollution effects over a century on the benthic marine algae of Co. Durham, England. J Linn Soc, London, Bot 70:269-305

Fairweather PG (1990) Sewage and the biota on seashores: assessment of impact in relation to natural variability. Environ Monit Asses 14:197-210

Golubic S (1970) Effect of organic pollution on benthic communities. Mar Pollut Bull 1:56-57

Grigg RW, Kiwala RS (1970) Some ecological effects of discharged wastes on marine life. Calif Fish Game 56: 145-155

Hagmeier E (1971) Turbidity - plants. In: Kinne O (ed) Marine ecology, Vol 1. Wiley, New York, p 1177-1180

John DM (1968) Studies on littoral and sublittoral ecosystems. PhD thesis, University of Durham

Jongman RH, ter Braak CJF, van Tongeren OFR (1987) Data analysis in community and landscape ecology. Pudoc, Wageningen

Kent M, Coker P (1992) Vegetation description and analysis A practical approach. Belhaven Press, London

Klavestad N (1978) The marine algae of the polluted inner part of the Oslofjord. Botanica mar 21:71-97

Littler MM, Murray SN (1975) Impact of sewage on the distri-

This article was submitted to the editor bution, abundance and community structure of rocky intertidal macroorganisms. Mar Biol 30:277-291

Lundälv T, Larsson CS, Axelsson L (1986) Long-term trends in algal-dominated rocky subtidal communities on the Swedish west coast - a transitional system? Hydrobiologia 142:81-95

May V (1985) Observations on algal floras close to two sewage outlets. Cunninghamia 1:385-394

Munda IM (1980) Changes in the benthic algal associations of the vicinity of Rovinj (Istrian coast, North Adriatic) caused by organic wastes. Acta Adriat 21:229-332

Murray NS, Littler M (1977) Seasonal analyses of standing stock and community structure of macroorganisms. In: Littler MM, Murray NS (eds) Influence of domestic wastes on the structure and energetics of intertidal communities near Wilson Cove, San Clemente Island. Tech Compl Rep 164, California Water Resources Center, Davis, p 7-32

Murray NS, Littler M (1978) Patterns of algal succession in a perturbed marine intertidal community. J Phycol 14 $506-512$

Podani J (1993) SYN-TAX - computer program for multivariate data analysis in ecology and systematics (version 5.0). Scientia Publishing, Budapest

Rai LC, Gaur JP, Kumar HD (1981) Phycology and heavymetal pollution. Biol Rev 56:99-151

ter Braak CJF (1988) CANOCO - a FORTRAN program for canonical community ordination by (partial) (detrended) (canonical) correspondence analysis (version 2.1). TNO Institute of Applied Computer Science, Wageningen

Manuscript first received: July 25, 1994

Revised version accepted: June 12, 1995 